Japan. J. Med. Sci. Biol., 1985

\title{
2. Summary of Reported Isolations of Bacteria, 1984
}

Overview

The number of reports of isolations of bacteria involving imported cases in 1984 (Fig.5) were as follows: 17,835 at PHI/HC, 67,723 at GCI, 1,191 at IDH, and 1,628 at quarantine stations. The respective figures represent $93 \%, 143 \%, 88 \%$ and $85 \%$ of the reported number in the year before. The numbers therefore, except at GCI, are smaller than the preceding year. The outstanding increase in the reports from GCI is considered to be due to the further improvement of the information collection system.

The number of isolation from overseas travelers was 2,453 at $\mathrm{PHI} / \mathrm{HC}$ (13.8\% of total isolations from the respective institution), 38 at GCI $(0.06 \%), 430$ at IDH (36.1\%), and 1,628 at quarantine stations (100\%). The proportion at $\mathrm{PHI} / \mathrm{HC}$ increased slightly compared with that of the year before.

For $\mathrm{PHI} / \mathrm{HC}$, the bacterial patohgen reported most frequently was Salmone ZZa, accounting for $23.8 \%$ of all PHI/HC reports in 1984 (25.2\% in 1983). Other bacterial pathogens found were as follows: Streptococcus accounting for 20.9\% (20.4\% in 1983), Campylobacter jejuni/coli $13.5 \%$ (13.4\%), Vibrio parahaemolyticus 12.4\% (10.9\%) and Escherichia coli $9.9 \%$ $(9.2 \%)$. The order of frequency was the same as the year before. The breakdown of cases of isolations from overseas travelers, namely imported cases, were: $32.3 \%$ for E.coli (29.9\% in 1983), $24.4 \%$ for Salmonella (31.2\%), $13.7 \%$ for V.parahaemolyticus (10.4\%), 9.9\% for Shigella (12.5\%), $6 \%$ for Vibrio cholerae $0-1$ and non $0-1$ (3.6\%), 5.4\% for Plesiomonas shigelloides (5.0\%) and $5.2 \%$ for $C . j e j u n i / c o l i(5.3 \%)$. The types of major bacteria remained unchanged but the percentages of Salmonella and Shigella decreased while the percentages of E.coli and V.parahaemolyticus increased (Fig.5-1).

$\mathrm{GCI}$, in contrast to $\mathrm{PHI} / \mathrm{HC}$, reported more isolations of bacterial pathogens from the respiratory tract than from the intestinal tract. The percentages of reports for these respiratory pathogens in 1984 were $22.7 \%$ for Streptococcus (22.5\% in 1983), 22.3\% for Haemophilus influenzae (19.9\%), $20.0 \%$ for Klebsiella pnezmoniae (22.9\%) and $13.4 \%$ for Streptococcus pneumoniae (11.9\%). As for bacteria causing infectious diseases of the gastro-intestinal tract, the percent breakdown of all reports were $7.9 \%$ for C.jejuni/coli (9.4\% in 1983), $2.6 \%$ for SalmoneZZa (3.6\%) and $3.1 \%$ for E.coli (2.3\%). 
Vol. 38, Suppl.
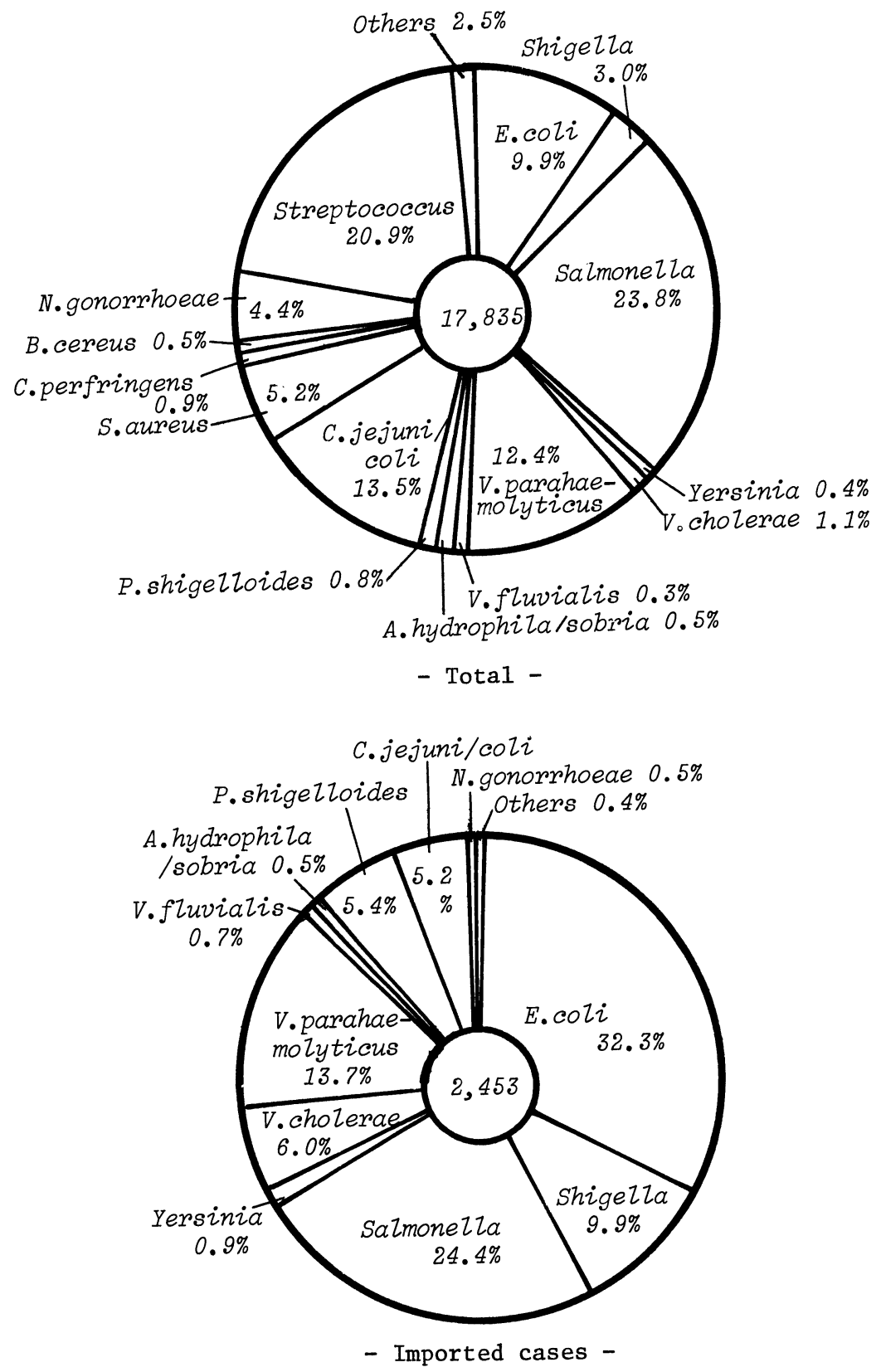

Fig. 5. Reported isolations of bacteria from humans, 1984.

5-1. Isolations by prefectural and municipal public health institutes and health centers. 
Japan. J. Med. Sci. Biol., 1985
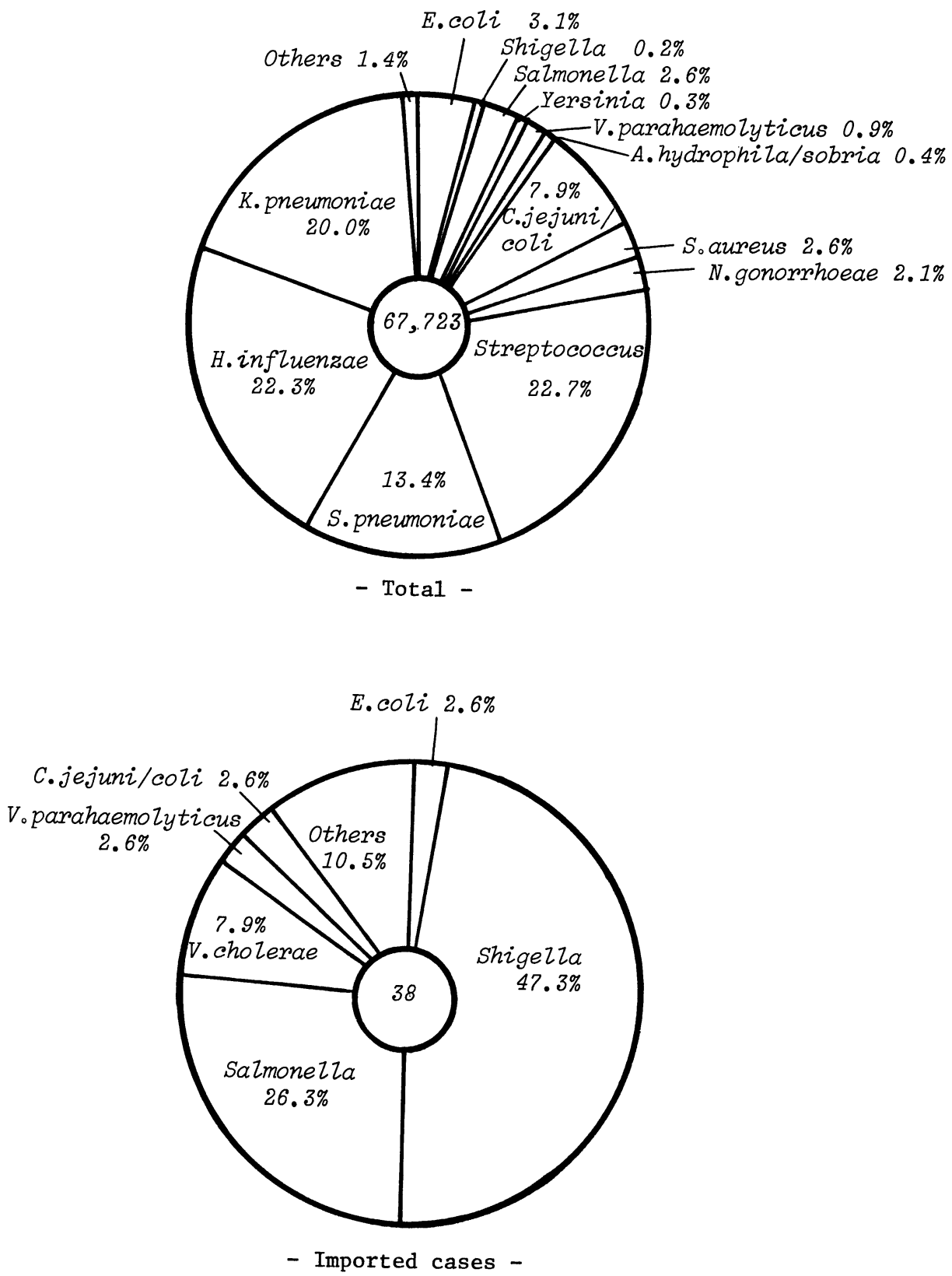

5-2. Isolations by general clinical institutions. 


\section{Vol. 38, Suppl.}

Thirty-eight imported cases were reported, the majority of which were intestinal pathogens. Of these, 18 cases (47.4\%) were Shigella (Fig.5-2).

The most frequent enteric pathogens isolated at quarantine stations were Salmonella, P.shigelloides, and V.parahaemolyticus. There were only a few isolations of enteropathogenic $E$.coli because the detection of these bacteria are not performed routinely. For all other gastro-intestinal pathogen isolations, quarantine station frequencies were almost equal to those of imported cases reported from PHI/HC.

\section{Shigella}

The annual number of Shigella reported by PHI/HC was 531 in 1984 . The reported number of Shigella ranged between 500-600 in 1979, 1980, and 1982. However, the number rised to 815 in 1981, and was 1,180 in 1983, more than twice the expected annual incidence. Outbreaks in 1984 reverted to the usual annual level (Fig.6-1). Of the total Shigella findings, there were 322 of S.sonnei (60.6\%), 184 of S.flexneri (34.7\%), 14 of S.boydii $(2.6 \%)$, and 11 of $S . d y s e n t e r i a e(2.1 \%)$. The number of imported cases of Shigella in 1984 was 245 (46.1\%). These cases accounted for $50 \%$ of S.sonnei, $35 \%$ of S.flexneri, $91 \%$ of S.dysenteriae and $71 \%$ of S.boydii. Monthly isolations of Shigella from 1980 to 1984 are shown in Fig.6-2, and number of isolations by group are shown in Fig.6-3.

At IDH, 436 detections of Shigella was reported in 1984. This number is less than the previous year's 603 detections, but is similar to the 1982 level (Fig.6-1). Two hundred twenty three imported cases were reported amounting to $51.1 \%$ of the total. Among these, $4.5 \%$ were S.dysenteriae, $38.6 \%$ were S.flexneri, $3.1 \%$ were S.boydii and $53.8 \%$ were S.sonnei. These rates were consistent with past trends.

At quarantine stations, 165 detections were reported, of which S.dysenteriae, S.flexneri, S.boydii and S.sonnei represented $1.2 \%, 29.7 \%, 6.7 \%$ and $62.4 \%$, respectively.

\section{Salmonezza}

$\mathrm{PHI} / \mathrm{HC}$ reported a total of 4,237 isolations of SalmonelZa from humans involving S.typhi and S.paratyphi. This was $88 \%$ of the previous year's total. Among these isolations, there were 559 imported cases which was $14.1 \%$ of the total. Reported findings by month rise to a broad peak in the 
Japan. J. Med. Sci. Biol., 1985

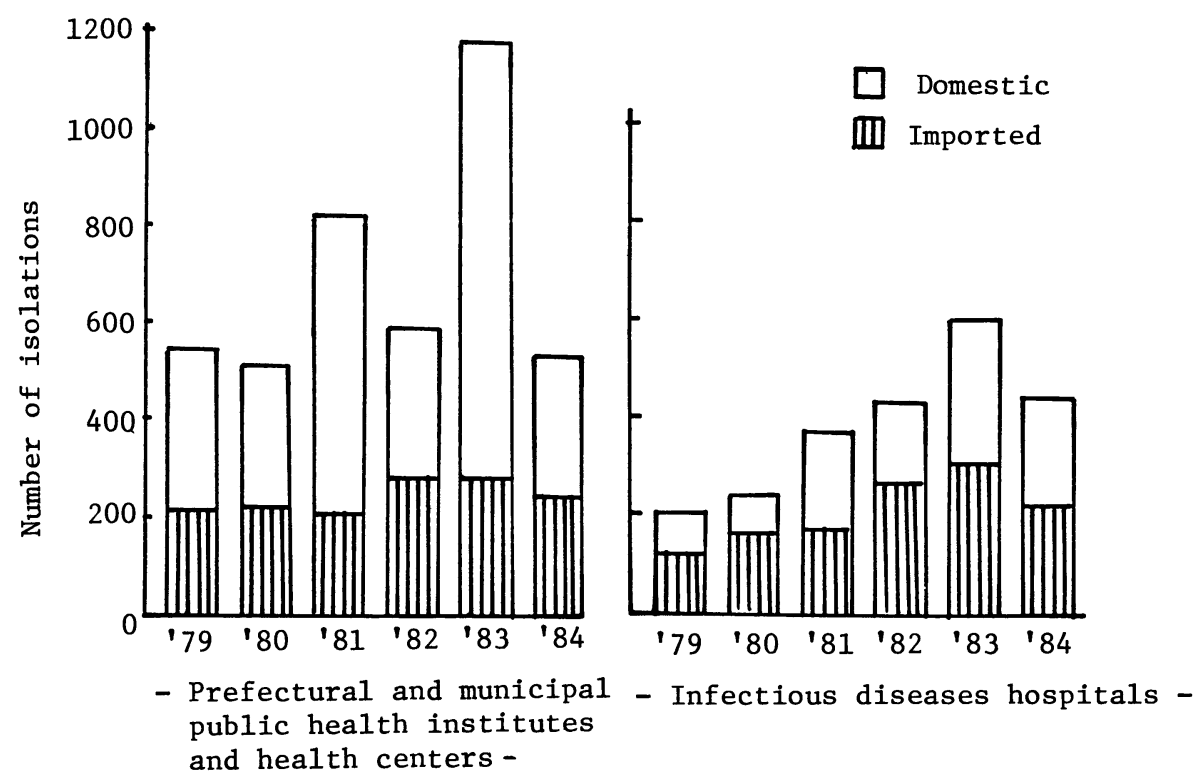

Fig. 6. Reported isolations of Shigella from humans, 1979-1984.

6-1. Isolations by prefectural and municipal public health institutes and infectious diseases hospitals, by year.

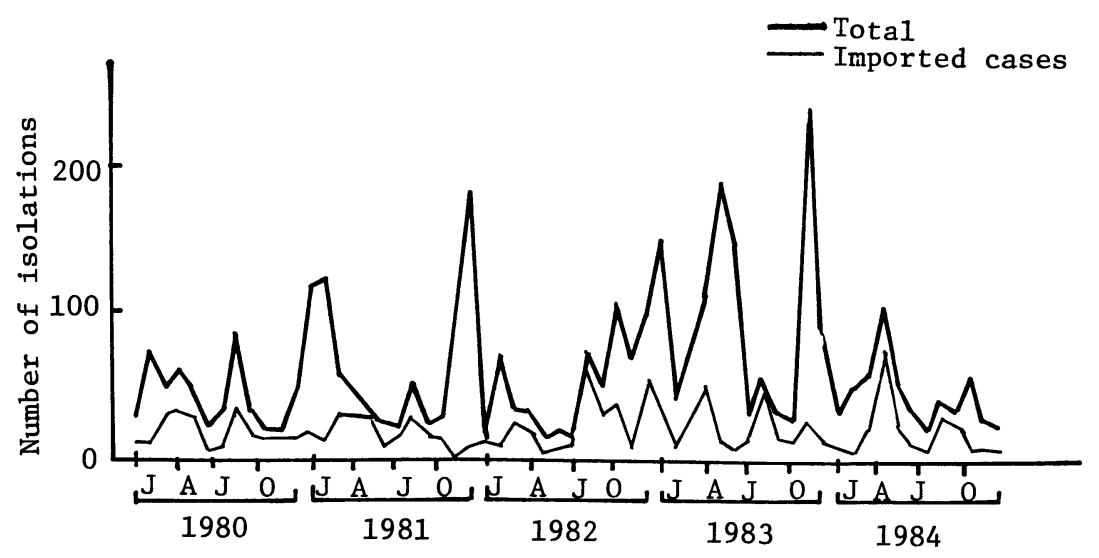

6-2. Isolations by prefectural and municipal public health institutes and health centers, by month. 
Vol. 38, Suppl.

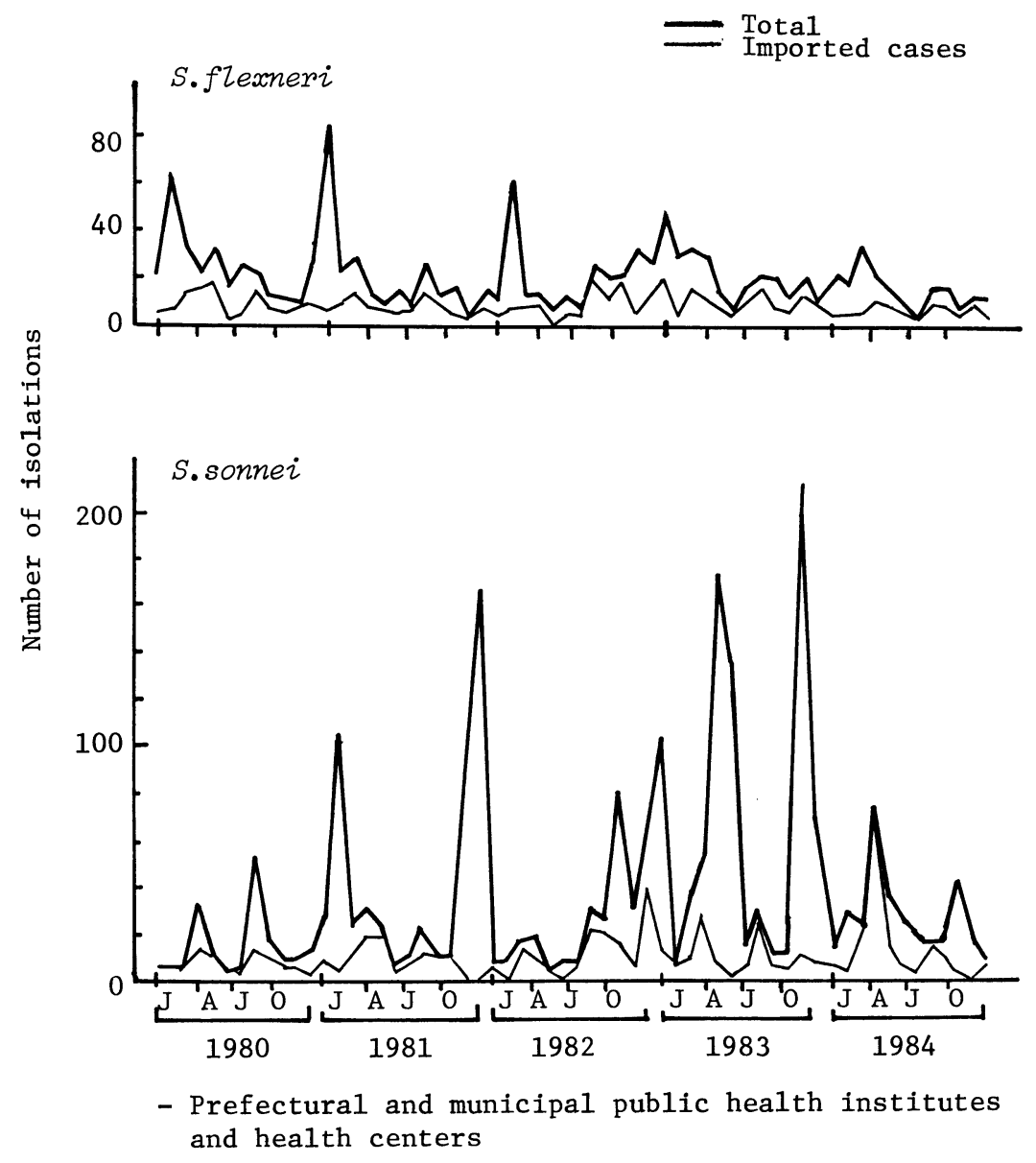

6-3. Isolations of S. flexneri and S. sonnei, by month.

summer, centered around August-September, on a yearly basis (Fig.7).

The main reason for the peak is the high frequency of outbreaks of food poisoning during this season. Apart from that, another important factor which increased the reported number is that results of the periodic fecal examination for food handlers are mainly submitted in this season. Findings of SaZmoneZZa reported by IDH are also highest in July-September.

In general, for all reports, the most frequently isolated SaZmoneZZa group was group B (including S.paratyphi B) (Fig.8). The group B frequencies for the respective institutions were: $36.3 \%$ at $\mathrm{PHI} / \mathrm{HC}$, and $51.8 \%$ at IDH. For group B, the number of imported cases from PHI/HC and quarantine stations were $27.4 \%$ and $28.6 \%$, respectively. The nest most frequent isolat- 


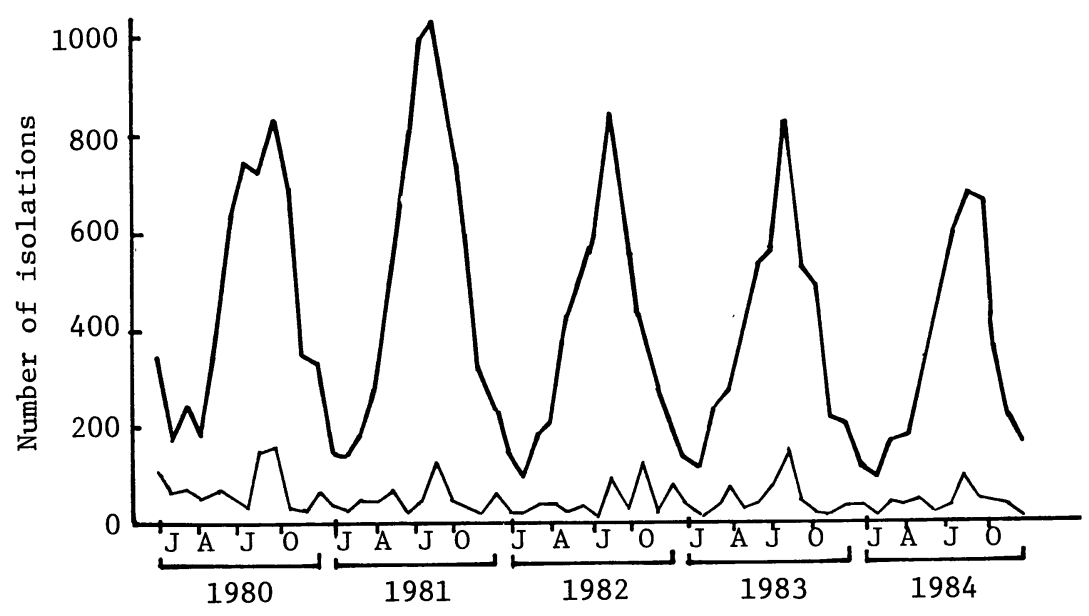

Fig. 7. Reported isolations of Salmonella other than S. typhi and S. paratyphi A from humans by prefectural and municipal public health institutes and health centers, by month, 1980-1984.

ions were group $\mathrm{C} 1$ and then group $\mathrm{C} 2-\mathrm{C} 3$. Imported cases, however, demonstrated a relatively higher frequency of group E1 and E4; the group E1 and E4 frequencies of all imported SaZmoneZZa were $28.3 \%$ at quarantine stations and $22.9 \%$ at $\mathrm{PHI} / \mathrm{HC}$. In contrast, group E1 and E4 SaZmoneZZa represented only $6.7 \%$ of domestic SalmonelZa detections reported from PHI/HC.

Among the SalmonelZa derived from humans, that was investigated at PHI/ HC, serovars were reported for 4,212 strains. For all SaZmoneZZa serovars tested, 15 comprised the majority and represented 72.8\%. Among them, 669 S.typhimurium represented the overwhelming majority (15.9\%) of all the serovar isolations, followed by 444 S.litchfield (10.5\%), 368 S.paratyphi B $(8.7 \%)$, and 265 s.infantis (6.3\%) (Fig.8-3). These serovar frequencies are consistent with previous trends.

Concerning the nomenclature of the SalmonelZa 0 serologic group, the Salmonelza Subcommittee of the International Enteric Bacterial Committee has determined that the previous Kauffmann-White Scheme should be changed. The change was recommended and finalized December 1984. Under the new taxonomic system, some serovars were deleted and some were combined. The reason for this change is that the number of SalmoneZZa serovars exceeded 1,600, and some of the 0 antigen is transmitted by phage making the 0 antigen express- 
Vol. 38, Suppl.

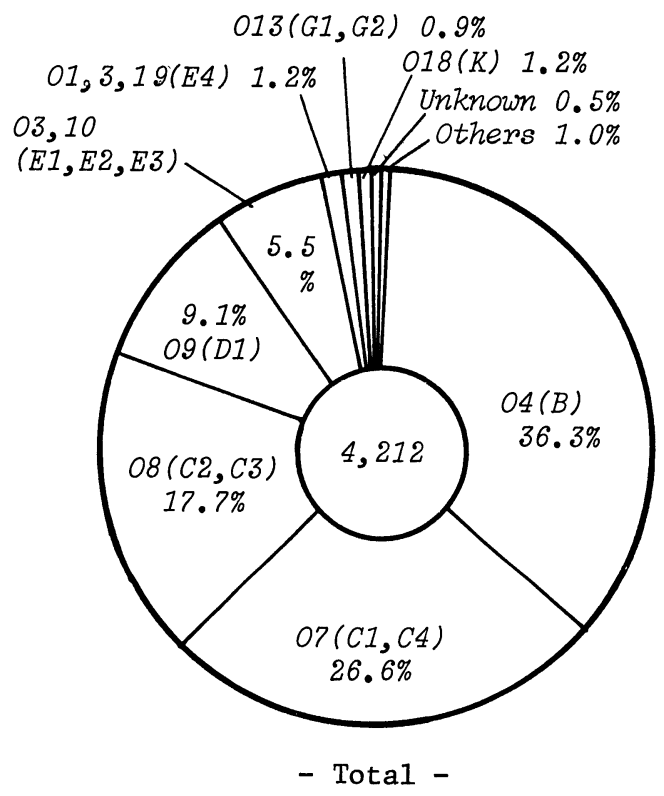

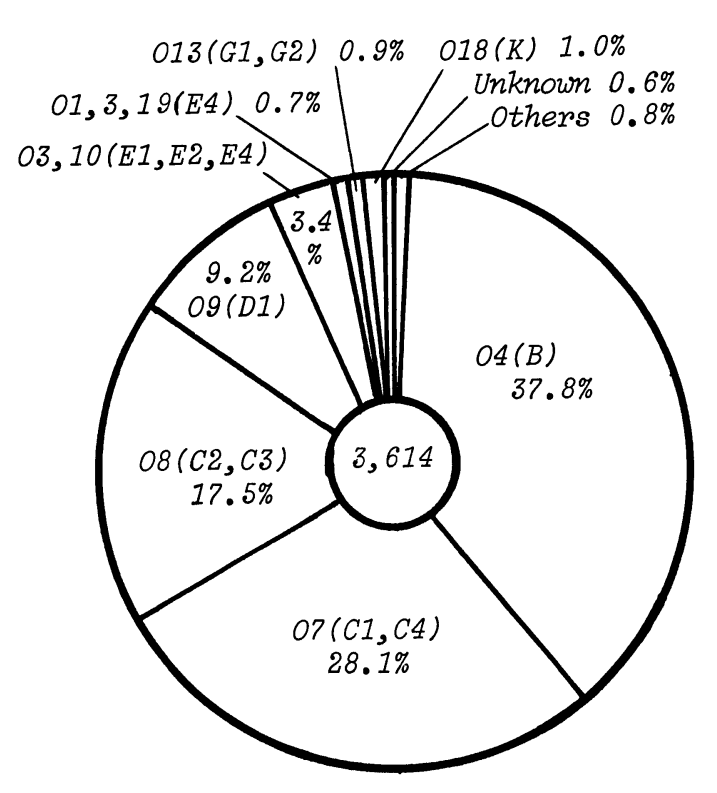

- Domestic -

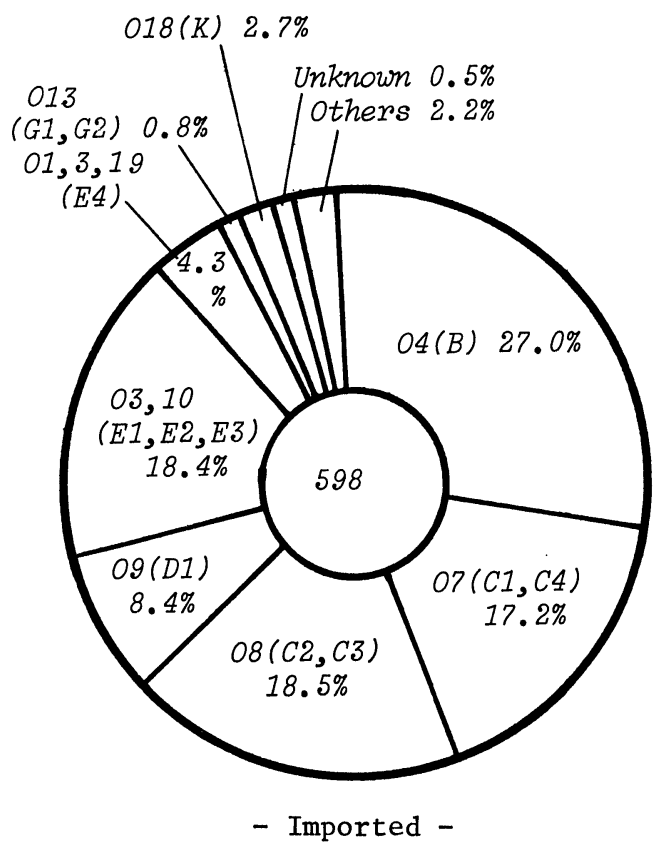

Fig. 8. Salmonella groups isolated by prefectural and municipal public health institutes and health centers, 1984.

8-1. Isolations from humans. 

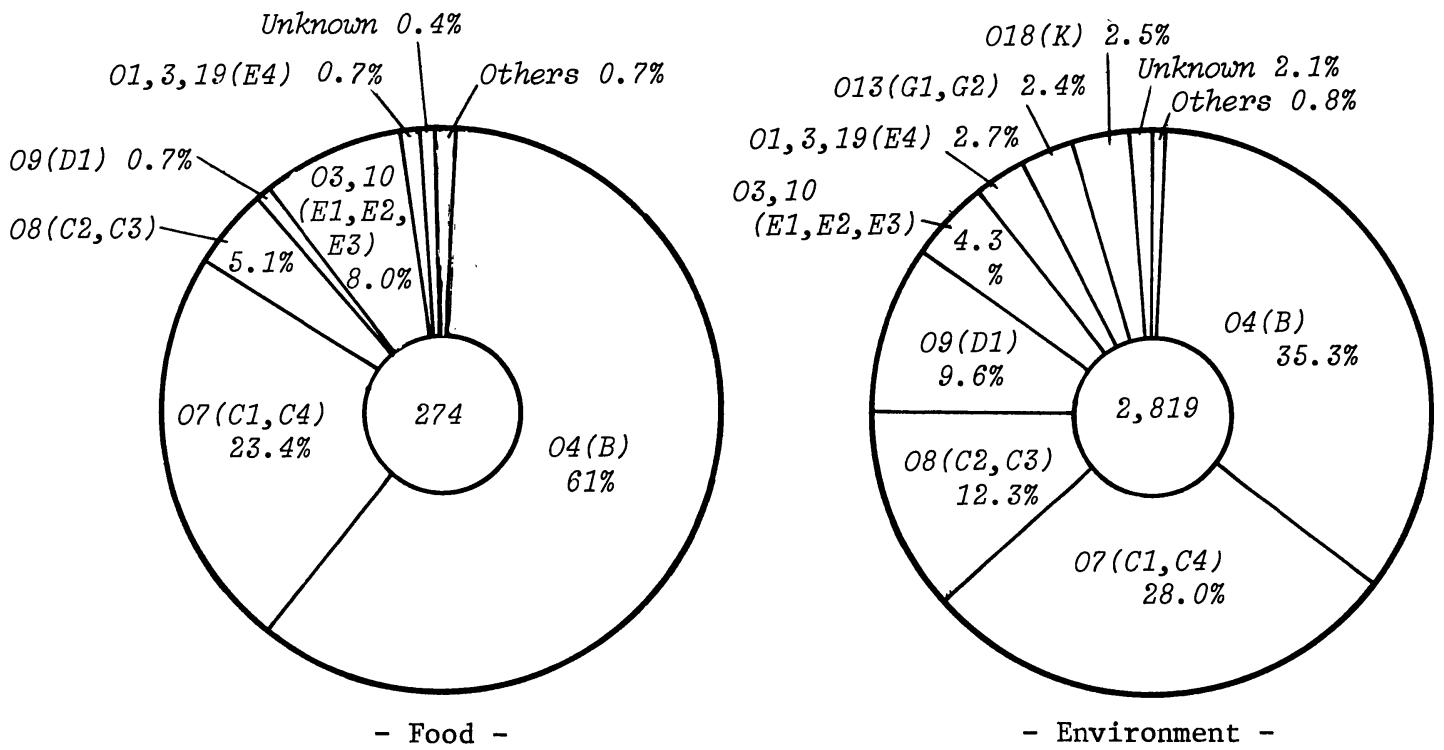

8-2. Isolations from food and environment.

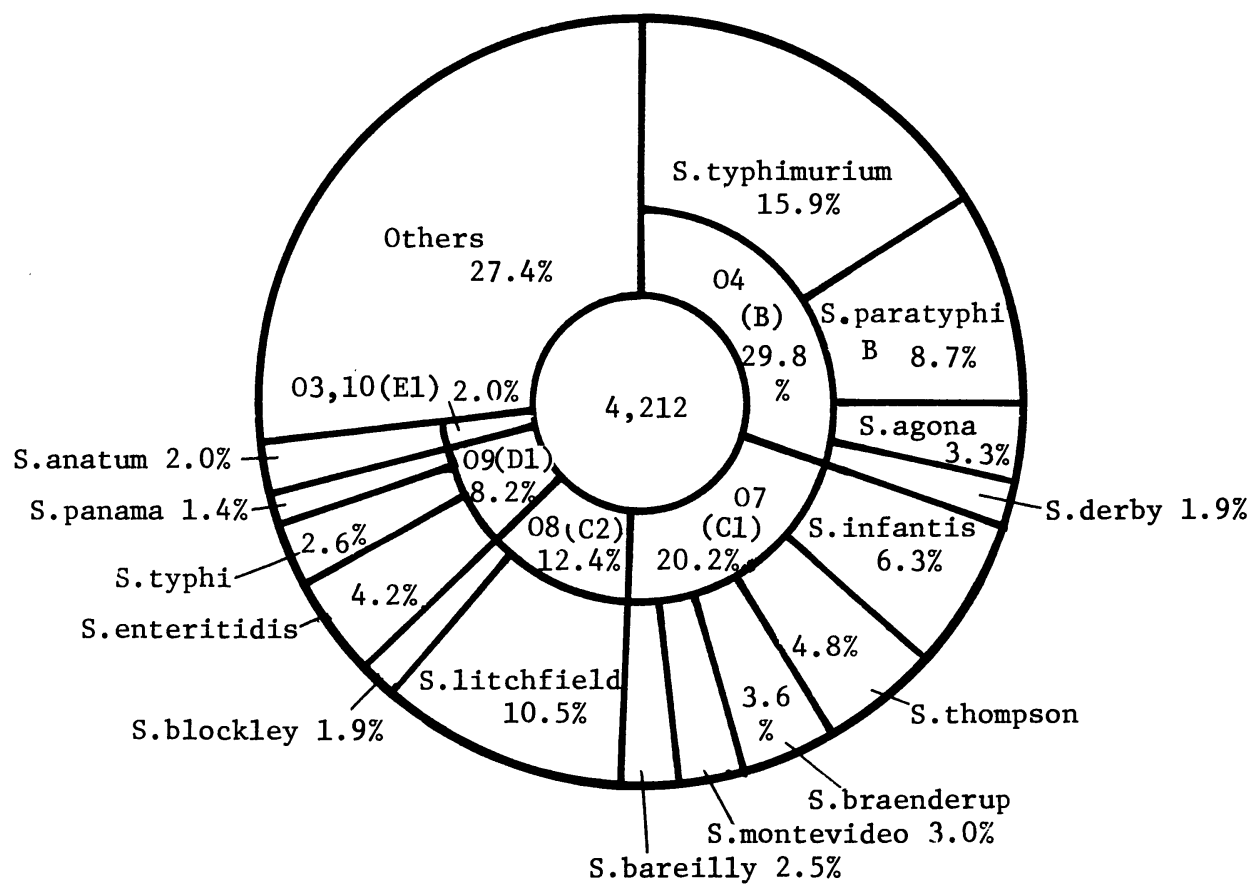

8-3. Fifteen most common Salmonella serovars from humans. 
Vol. 38, Suppl.

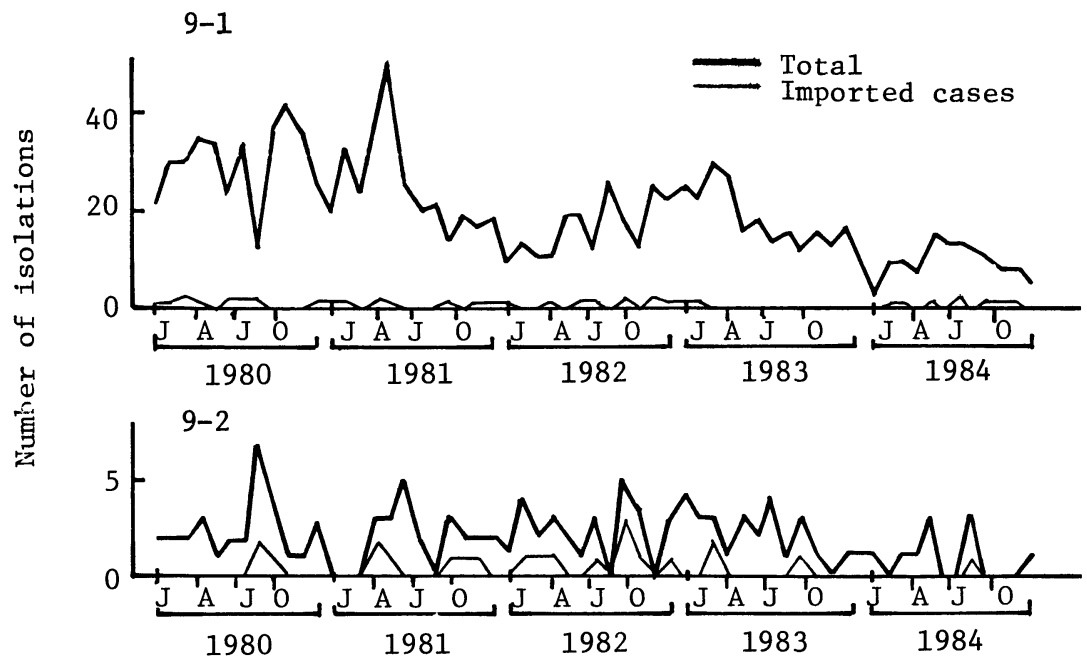

Fig. 9. Reported isolations of $S$. typhi and $S$. paratyphi $A$ from humans by prefectural and municipal public health institutes and health centers, by month, 1980-1984.

9-1. Isolations of S. typhi.

9-2. Isolations of $S$. paratyphi $A$.

ion unstable. For the present report, although Salmonella monthly reports (Table I, II) are tabulated according to the old scheme, the serovar data (Table III) was changed to the new nomenclature via computer programming.

\section{S.typhi, S.paratyphi A and S.paratyphi B}

Among SalmonelZa described above, reported findings of $S . t y p h i$ totalled 110 isolations (with eight imported cases) at PHI/HC. This was about half as many as last year. There were 10 isolations of $S$.paratyphi $A$ reported (two imported) and 312 isolations of S.paratyphi B (four imported) at PHI/ /HC. IDH reported: 62 S.typhi (21 imported), 14 S.paratyphi A (eight imported), and $37 \mathrm{~S}$.paratyphi $B$ (one imported). No seasonal trends are detected for S.typhi or S.paratyphi A. However, isolations of S.paratyphi B increased in the summer, as did many other Salmonella, and peaked in August (Fig.9-1, 9-2).

Reports of S.typhi, and S.paratyphi $A$ and $B$ submitted in accordance with a notice from the Bureau of Public Health represented the total number of cases of typhoid and paratyphoid fevers in Japan on a national scale. For patients and carriers in 1984, there were 228 cases of S.typhi ( 47 imported), 28 cases of S.paratyphi $A$ (14 imported) and 204 cases of 
S.paratyphi B (one imported). Among these isolations those which were submitted for phage typing accounted for $93 \%$ of S.typhi, all of S.paratyphi $A$ and all except three strains of S.paratyphi $B$.

For S.typhi, 22 phage types were isolated, of which types D2, DVS (Degraded Vi positive strains), $\mathrm{M} 1$ and $\mathrm{E} 1$ were reported frequently and ranged throughout the country. These phage type patterns were similar to 1983. There were 15 phage types of S.typhi from overseas travellers, among which, C1, E2, 0, 40, 53-1 were found. Interesting1y these phage types were not isolated from any domestic cases.

For S.paratyphi A, six phage types were isolated. Most frequently detected were: untypable, followed by type 1, and type 4. For S.paratyphi $B$, eight phage types were isolated and one isolation from an imported case was untypable.

Drug sensitivities were conducted on all the isolated strains submitted for phage typing. One $S . t y p h i$ proved resistant, and was isolated from an imported case. It was found to be type M1 (resistant to SM.ABPC).

\section{Enteropathogenic E.coli}

In this surveillance system, $E . c o l i$ isolation reports were limited to those considered as a cause of diarrhea. For PHI/HC in 1984, isolations numbered 1,766; that is about the same as last year. Imported cases accounted for 792 cases (44.8\%) showing a slight increase in comparison with last year's, 664 cases. Enteropathogenic E.coli findings reported by PHI/HC, increased mainly in the summer reflecting outbreaks of food poisoning caused by this bacteria. In 1984, the peak incidence was in July and August (Fig. 10-1). However, the number of E.coli reports from GCI does not increase in summer season (Fig.10-2).

1,626 E.coli isolations reported from PHI/HC in 1984 were further tested. These were categorized by pathogenicity as follows; $6.2 \%$ enteroinvasive, $54.9 \%$ enterotoxigenic, and $38.9 \%$ enteropathogenic serotype. Among the 792 imported cases, 727 were further tested, and the most frequent $E$. coli was enterotoxigenic representing $64.6 \%$.

\section{V.parahaemolyticus}

In 1984, PHI/HC reported 2,207 isolations of which 337 were imported 
Vol. 38, Suppl.
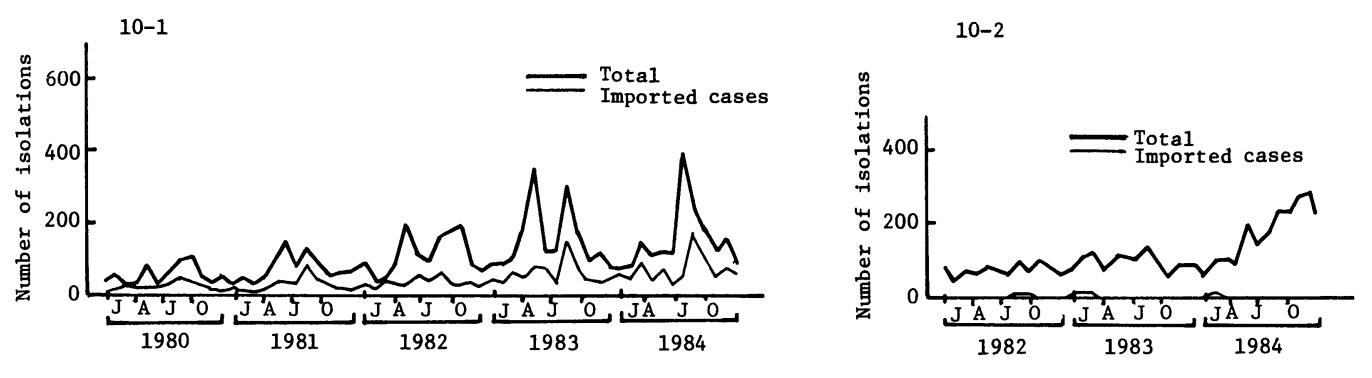

Fig. 10. Reported isolations of $E$. coli from humans, by month, 1980-1984.

10-1. Isolations by prefectural and municipal public health institutes and health centers.

10-2. Isolations by general clinical institutions.
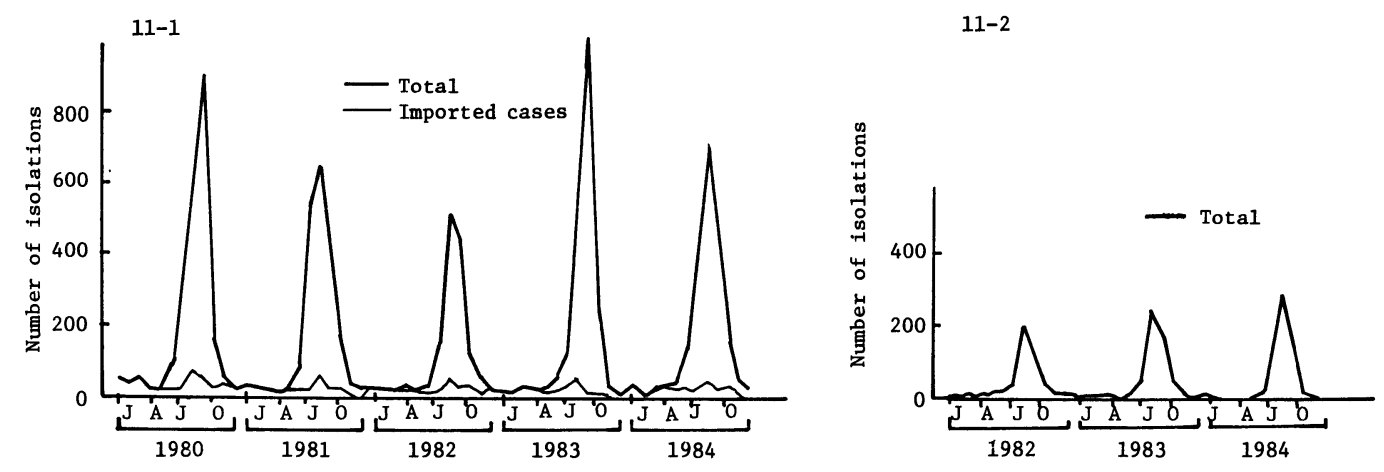

Fig. 11. Reported isolations of V. parahaemolyticus from humans, by month, 19801984.

11-1. Isolations by prefectural and municipal public health institutes and health centers.

11-2. Isolations by general clinical institutions. 
cases. These numbers accounted for $106 \%$ and $145 \%$ of last year's figures, respectively. Reports were concentrated in the summer with the peak in August, reflecting large-scale outbreaks of food poisoning caused by this bacteria (Fig.11).

Isolations from IDH totaled 90, with 21 imported cases. There were 293 cases from quarantine stations. These figures represent $80-90 \%$ of the total reported in the last year.

\section{C.jejuni/coli}

In this surveillance system, only findings of $C . j e j u n i$ and $C$. coli which caused diarrhea were tabulated among Campylobacter isolations. The number of reports in 1984 was 2,400 (involving 127 imported cases) at PHI/HC, 5, 376 (one imported) at GCI, and 146 (25 imported) at IDH, representing 93.8\%, $121.1 \%$, and $94.2 \%$ of the number reported in 1983 , respectively. Many outbreaks of enteritis by Campylobacter were caused by school lunch, and concentrated in the earlier part of the food poisoning season, May and June (Fig.12).

C.jejuni/coli were reported in considerably greater numbers at GCI than at $\mathrm{PHI} / \mathrm{HC}$, which is a characteristic of C.jejuni/coli different from the other intestinal bacteria. This is probably due to the more frequent diagnostic assays in GCI performed in patients infected with this organism as the clinical symptom is relatively severe especially in the young children. Since considerable numbers of findings are reported not only in summer season but also in any other season by GCI, numerous sporadic cases occur al1 through the year.

\section{Other Bacteria Associated with Gastro-intestinal Diseases}

V.fluvialis, V.mimicus, Aeromonas hydrophila/sobria, and P. shigelloides were added to the report form when these bacteria were considered as a cause of food poisoning. The legal notification was issued by the chief of the Section of Food Hygiene of the Ministry of Health and Welfare (Publication 非59 of Environment and Food: "For the handling of NAG Vibrio, Campylobacter and so on food hygiene" on 11th of March, in 1982). For these species, the isolations earlier than 1982 were reported as "Other bacteria". In 1984, the number of isolations at PHI/HC was: 55 V.fluvialis (18 imported cases), 14 V.mimicus (none imported), 83 A.hydrophila/sobria (11 imported), and 140 
Vol. 38, Suppl.
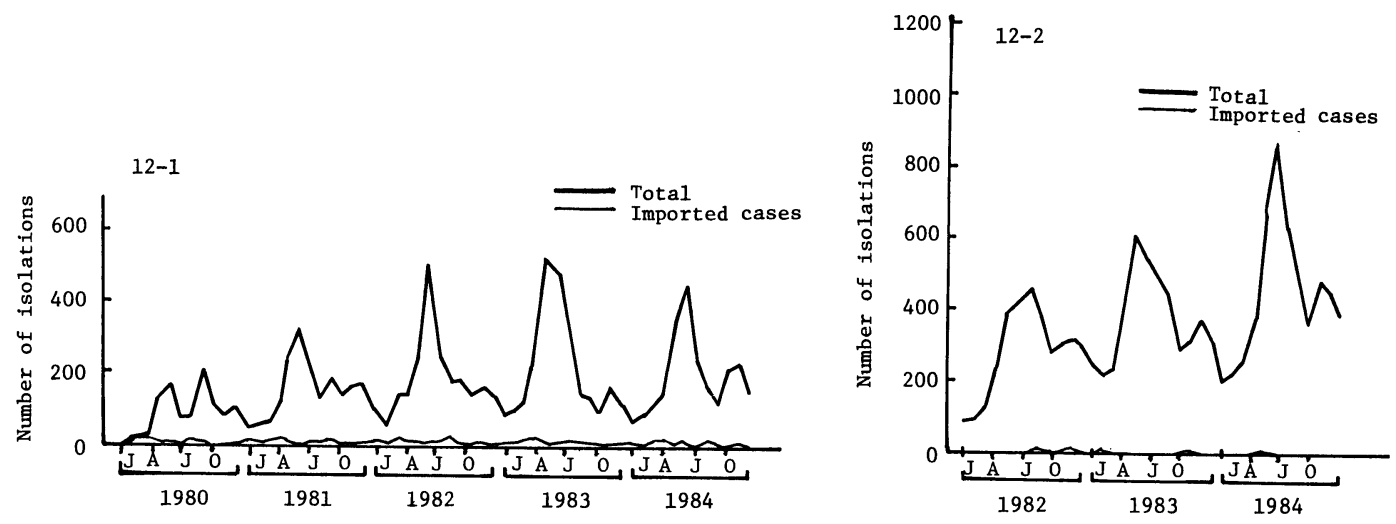

Fig. 12. Reported isolations of C. jejuni/coli from humans, by month, 1980-1984.

12-1. Isolations by prefectural and municipal public health institutes and health centers.

12-2. Isolations by general clinical institutions.
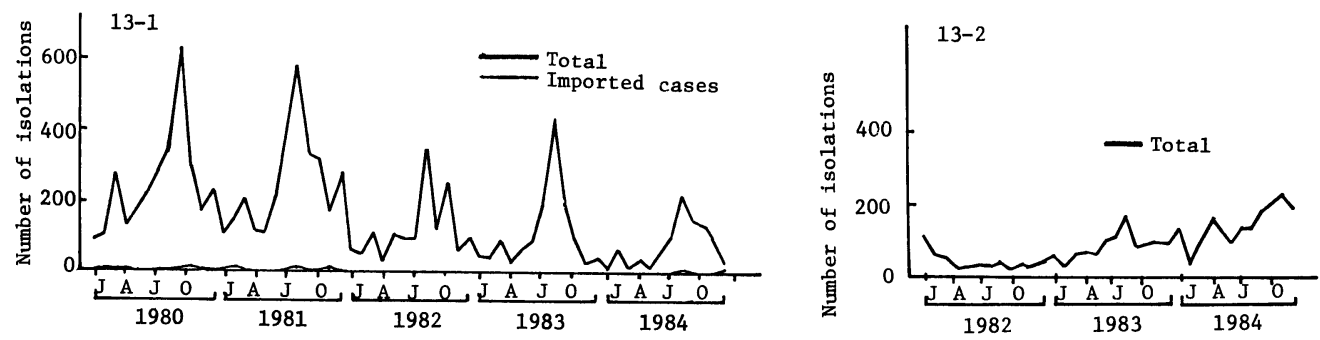

Fig. 13. Reported isolations of $S$. aureus from humans, by month, 1980-1984.

13-1. Isolations by prefectural and municipal public health institutes and health centers.

13-2. Isolations by general clinical institutions. 
P.shigelloides (133 imported). These numbers represented 73.3\%, 700\%, 250\% and $109 \%$ respectively of the number reported in 1983. For P.shigelloides, a majority of the isolations were imported cases and 342 isolations at quarantine stations represented $21.0 \%$ of the total number of findings reported from quarantine stations.

In 1984, reported number of isolations of Staphylococcus aureus (Fig. 13), Yersinia enterocolitica and Clostridium perfringens were 925 (two imported), 75 (21 imported) and 167 (none imported) from $\mathrm{PHI} / \mathrm{HC}$, respective$1 y$, and 1,770, 187 and seven from GCI (none imported for these bacteria), respectively. In addition, seven isolations of C.botulinum type A and three of C.botulinum type $\mathrm{E}$ associated with food-poisonings were reported.

\section{Streptococcus}

In 1984, PHI/HC reported 3,713 isolations of Streptococcus, while GCI reported 15,359 isolations. The overwhelming majority of isolations in both reports were of group A, accounting for $78.2 \%$ at PHI/HC and $52.9 \%$ at GCI. Streptococcus $A$ was tested for type at public health institutes. Of the 2,842 strains tested for type, the order of frequency of findings were $\mathrm{T}-12$ (24.0\%), T-4 (22.0\%), T-3 (14.4\%), followed by a much smaller percentage of $\mathrm{T}-28, \mathrm{~T}-1$, and $\mathrm{T}-13$. Compared with the frequency of last year, the frequency of $\mathrm{T}-13$ decreased and that of $\mathrm{T}-3$ increased. Peaks in January-March and November-December were seen for all types (Fig.14).

\section{Other Bacteria Associated with Respiratory Illness}

GCI submitted many reports of bacterial pathogens associated with respiratory illness, such as K.pneumoniae, H.influenzae and S.pneumoniae, of which detections in 1984 numbered 13,559, 15,105, and 9,080, respectively. Isolations of these three species increased and represented $120-160 \%$ of last year's figures. 
Vol. 38, Suppl.

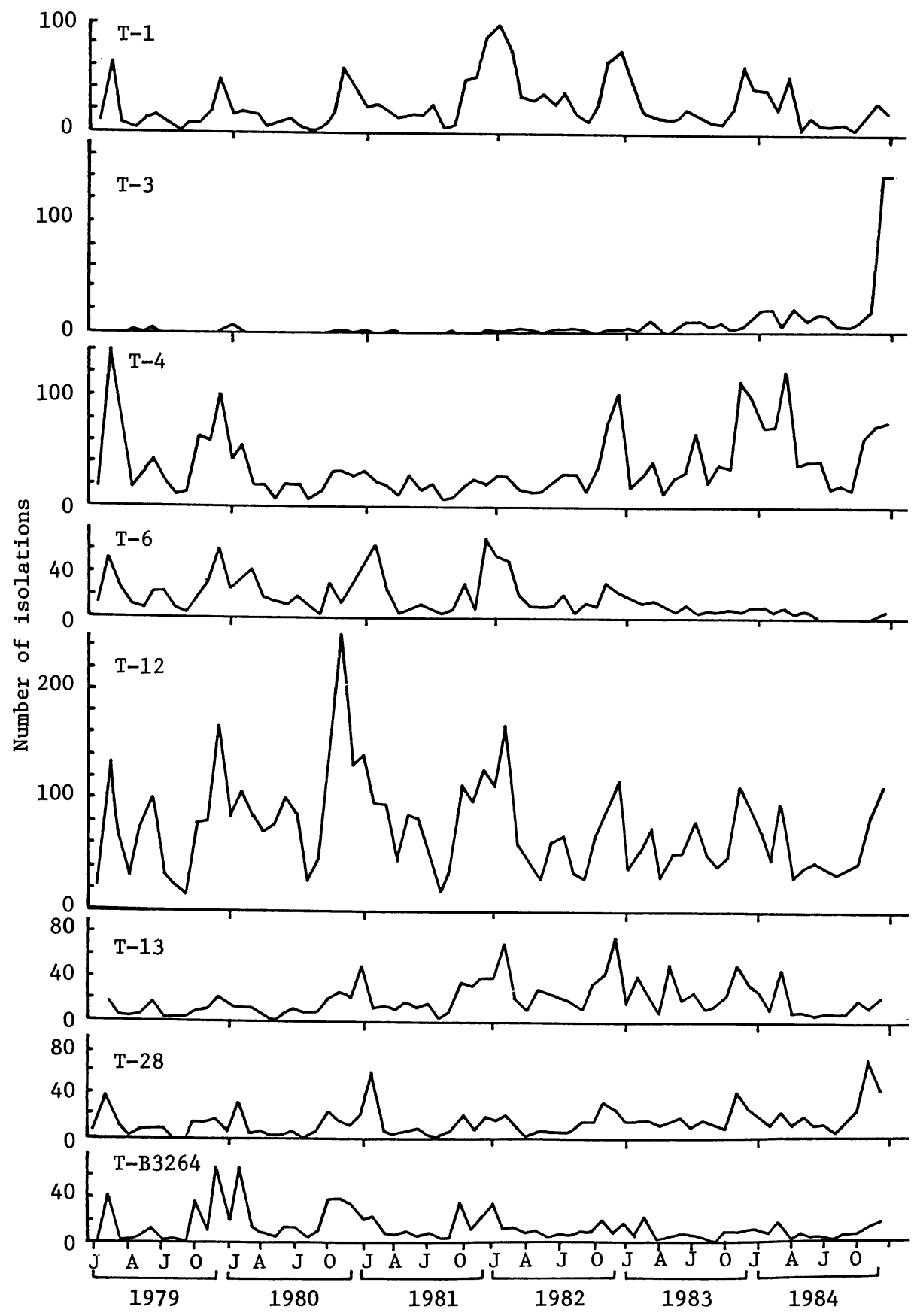

Fig. 14. Reported isolations of Group A Streptococcus serotypes from humans by prefectural and municipal public health institutes and health centers, by month, 1979-1984. 